
internationales

vol. 22 - $n^{\circ} 3 \mid 2006$

Turquie 2006 : aux portes de l'Union européenne ?

\title{
Entre ville et désert : Mobilité, activités et urbanité dans l'espace Sahara-Sahel
}

Compte rendu d'un colloque international au Centre de l'Orient Moderne (ZMO) à Berlin les 10-12 décembre 2005

Elisabeth Boesen et Laurence Marfaing

\section{(2) OpenEdition}

Journals

Édition électronique

URL : https://journals.openedition.org/remi/4122

DOI : $10.4000 /$ remi. 4122

ISSN : $1777-5418$

Éditeur

Université de Poitiers

Édition imprimée

Date de publication : 31 décembre 2006

Pagination : 253-258

ISBN : 978-2-911627-43-9

ISSN : 0765-0752

Référence électronique

Elisabeth Boesen et Laurence Marfaing, « Entre ville et désert : Mobilité, activités et urbanité dans

l'espace Sahara-Sahel », Revue européenne des migrations internationales [En ligne], vol. 22 - n³ | 2006, mis en ligne le 31 décembre 2009, consulté le 16 avril 2022. URL : http://journals.openedition.org/ remi/4122; DOI : https://doi.org/10.4000/remi.4122

Ce document a été généré automatiquement le 16 avril 2022.

(C) Université de Poitiers 


\section{Entre ville et désert : Mobilité, activités et urbanité dans l'espace Sahara-Sahel}

Compte rendu d'un colloque international au Centre de l'Orient Moderne (ZMO) à Berlin les 10-12 décembre 2005

Elisabeth Boesen et Laurence Marfaing

1 Pour percevoir les phénomènes « d'installation », c'est-à-dire les institutions sociales et les formes d'organisation qui sont issues des mouvements, nous avons choisi de nous concentrer sur les espaces urbains et sur les manifestations locales des relations translocales, c'est-à-dire le quotidien de l'existence urbaine des migrants.

2 L'idée d'une «appropriation » de l'espace urbain par les migrants, repose en grande partie sur le fait que l'observation est concentrée sur les migrants de type classique, c'est-à-dire sur certaines communautés de commerçants qui, en situation de diaspora, créent des communautés résidentielles (comme les zongos par exemple) et qui parviennent en plus à « occuper » l'espace public, les rues, les places en y faisant leurs affaires. Nous voulions appréhender les migrants qui ne sont pas seulement des Wanderer potentiels mais qui vivent leur mobilité comme les "péripatétiques » par exemple (ou service nomads ; cf. Rao 1985 ; Berland 1979), des colporteurs qui s'opposent pour ainsi dire à leur «localisation » et dont les activités urbaines ne s'inscrivent que de manière très limitée dans l'organisation de l'espace urbain. Il s'agit souvent de femmes, de jeunes, mais aussi de membres des sociétés nomades et de groupes marginaux comme les descendants d'esclaves par exemple. L'existence urbaine de migrants et leur potentiel à créer des « localités » (ou des «translocalités») restaient donc pour nous à étudier.

3 L'observation de ce type de citadin nous permet de cerner plus exactement la Fremdheit (extranéité, étrangeté) comme un élément des relations urbaines. Les populations locales distinguent très souvent plusieurs catégories d'étrangers (cf. Fortes 1975). Nos propres études montrent également que les groupes et les processus d'interactions diffèrent en ce qui concerne les fondements et les conceptions de l'étrangeté et sa 
signification sociale. Il s'agit entre autre de voir si les groupes de migrants accèdent au statut de citadin, voire s'ils le désirent. La clarification et la comparaison de ce facteur de l'étrangeté sont aussi d'importance dans le cadre de l'analyse comparative du problème général de la relation entre urbanité et étrangeté - un problème qui a été abordé par Gervais-Lambony (2003) dans son étude sur Lomé et Harare.

4 Les journées d'étude étaient organisées en plusieurs panels thématiques. La première portait sur la mobilité, la sédentarisation ou l'installation et l'organisation spatiale en milieu urbain : Il s'agissait ici de conceptualiser la mobilité, et d'analyser l'évolution des notions locales de mobilité par rapport aux opportunités économiques, aux politiques, aux nouvelles technologies. La seconde portait sur les activités des migrants urbains : leur travail, les activités marchandes et les différents métiers qu'ils pratiquent. Une troisième approche thématique portait sur l'extranéité/Fremdheit et les différentes formes de marquage identitaire qui y sont liées; il s'agissait d'analyser la persistance, l'évolution ou la substitution des rapports sociaux et des modes d'expression traditionnels.

5 En ce qui concerne l'ère géographique, nous avions en vue l'espace Sahara Sahel. Notre approche de la ville était également très large : des centres urbains occidentaux aux grandes villes africaines comme Dakar ou Abidjan en passant par les camps de réfugiés du Sahara Occidental. De ce point de vue, la question était de savoir si les populations de l'espace Sahara Sahel montrent des formes de mobilité particulières et si elles ont également des perceptions spécifiques de la " ville » ou de la " ville en devenir ».

6 Au début de notre colloque l'accent a été mis sur les notions conceptuelles : historique du terme de «migration» dans le contexte de la politique française de migration envers les habitants des anciennes colonies (Gregory Mann); perception qu'ont les différents acteurs des migrants et de la migration (Daouda Gary sur les migrants du Mali, du Burkina Faso et de la Côte d'Ivoire ; Abdoulaye Kane sur les pèlerins tijanes au Maroc) ; différenciations au sein d'une communauté migrante (Linda Beck par rapport à la communauté musulmane immigrée de New York).

7 Le deuxième jour a été consacré à la thématique suivante : «mobilité, sédentarisation, installation et organisation spatiale en milieu urbain ». L'organisation spatiale saharosahélienne se caractérise par une double bifurcation, entre d'une part l'urbain et le rural et d'autre part les modes de vie nomade et sédentaire. Ainsi les populations évoluent entre ces quatre pôles en fonction de leurs différentes, et souvent multiples, activités et leurs formes de production. Cette "condition saharo-sahélienne », cette dynamique entre nomadisme et sédentarité, se retrouve-t-elle dans le monde des migrants, c'est-à-dire dans les villes ouest-africaines et celles du Maghreb ? Existe-t-il des modes de vie que l'on pourrait qualifier de nomade dans l'espace urbain, et si oui, quelle influence exercent-ils sur l'organisation spatiale de la ville? À côté de formes historiques ou contemporaines d'une urbanité nomade - comme celle des quartiers nomades dans les centres villes dont l'architecture fait preuve d'un esprit d'installation éphémère - ne faut-il pas également considérer les nouvelles formes de mobilité comme celles des jeunes migrants ou des femmes migrantes qui ne bénéficient pas forcément des réseaux de solidarité ou qui s'en sont éloignés pour développer leurs propres formes de sociabilité. Pour répondre à ces questions, nous avons regardé des formes d'installations différentes dans des centres urbains différents : il s'agissait de celles des descendants d'esclaves migrants saisonniers à Abidjan (Florence Boyer), de celles des réfugiés sahraouis dans des camps autour de Tindouf (Sophie Caratini), de 
celles de migrants ouest africains à Tamanrasset (Dida Badi) et enfin de celles des commerçantes sénégalaises qui font des allers-retours entre Dakar et Casablanca (Laurence Marfaing).

8 Pour les femmes sahraouies de Tindouf, c'est la situation de guerre, et l'absence des hommes, qui devient vecteur de sédentarisation et de changement de l'ordre établi. Dans ce cas, l'accès à l'argent, notamment aux réseaux financiers internationaux, transforme les rapports de pouvoirs. Nous constaterons le même effet dans le contexte des développements agropastoraux de l'Air (André Bourgeot) ou de l'évolution de la propriété foncière chez les migrants du fleuve Sénégal (Jean Schmitz). Pour les commerçantes sénégalaises de Casablanca, c'est la religion et la représentation qu'a la société sénégalaise de la vie au Maroc, qui rend possible leur participation au commerce international et participe ainsi à une émancipation de l'ordre social sénégalais. À la différence de ces femmes commerçantes qui laissent les lieux de leurs activités inchangés en les quittant, les migrants maliens, burkinabé et nigérians à Tamanrasset, mettent en place des stratégies pour s'insérer dans l'économie locale. Pour les anciens esclaves ou les descendants d'esclaves de Bankilaré au Niger cependant, c'est avant tout la route qui s'avère être le lieu où les identités se transforment ; au retour ils évitent la confrontation avec leurs anciens maitres ou avec leurs aînés.

9 Le panel suivant avait pour titre: "Commerce, travail, activités et métiers ». La migration urbaine dans l'espace Sahara Sahel est souvent associée au commerce ou au pèlerinage. Ces formes d'activités urbaines sont cependant accompagnées de phénomènes spatiaux spécifiques dans la mesure où le quotidien, l'habitat et les activités liées au commerce forment bien souvent un tout (cf. Bredeloup 2003). Certains migrants, cependant, ont des activités qui ne permettent pas une installation, tels les marchands ambulants par exemple. Ils vivent souvent dans des situations précaires comme ces commerçantes Wodaabe qui passent chaque année plusieurs semaines voire des mois dans les rues de Bamako ou de Dakar (Elisabeth Boesen).

Un aspect important de ces activités diverses renvoie à de nouvelles perceptions de travail et de statut social. La question étant de savoir si elles sont partagées par les différents groupes? Ces nouvelles « relations de travail » se développent-elles à partir de relations anciennes? Historiquement, les tâches manuelles et l'artisanat étaient souvent accomplis par des populations descendant d'esclaves (Harratin, Iklan, Riimaaybe) et elles le sont encore dans une certaine mesure. Peut-on ici discerner deux conceptions de travail qui se définissent mutuellement : celle de ceux qui sont d'origine libre et celle de ceux qui sont d'origine servile? Le changement et la modernisation des relations économiques, leur intégration dans des échanges globaux laissent-elles apparaître des formes sociales que l'on pourrait caractériser d'urbaines? Ce processus a été illustré par les camps aurifères du Burkina Faso (Katia Werthmann) qui présentent une organisation quasi-urbaine où l'identité de la personne est limitée à sa force de travail. Ces camps, qui peuvent être caractérisés comme un monde masculin, offrent néanmoins des possibilités d'émancipation à une main-d'œuvre féminine, en leur permettant d'échapper aux mariages forcés.

11 Dans le secteur agropastoral, le passage d'une activité d'économie domestique à une activité marchande peut favoriser l'investissement dans l'immobilier et aboutir à une installation urbaine et une diversification dans le commerce (André Bourgeot). Paradoxalement les rendements permettront en même temps un re-investissement 
dans l'économie caravanière, notamment dans le commerce des pains de sel. L'on observe un processus semblable chez les Tuareg d'Agadez, devenus urbains par les aléas climatiques ; c'est le tourisme qui les rendra aux activités mobiles (chauffeurs, guides) et à leur statut social nomade de sorte que la ville est devenue pour eux, périphérie (Marco Scholze). Double paradoxe que celui de constater qu'Agadez justement, est intégré au patrimoine mondial grâce à son urbanisme soudanien, et n'est que le point de chute d'un tourisme international venu chercher la vie nomade.

Contrairement au constat d'une interaction entre population locale et migrante installée dans la ville de Tamanrasset (Dida Badi), l'investissement dans le commerce par les migrants saisonniers Songhay, s'il s'avère lucratif, mettra les acteurs à distance des populations locales (Isaïe Dougnon). Chez les Burkinabé et Maliens en Côte d'Ivoire (Daouda Gary), la perception de l'autre tout en bas de l'échelle sociale prime sur le facteur de production de richesses qu'il représente. Par contre ici aussi, comme pour les commerçantes sénégalaises à Casablanca, cette migration saisonnière rehausse le statut social dans la zone de départ. C'est également vrai pour les migrations saisonnières des Tuareg du Niger (Benedetta Rossi).

Ces problèmes de rapports de statut sont liés à la conception de l'étrangeté (Fremdheit) ou de la relation étranger/autochtone, thématique abordée dans le panel: «Extranéité/ Fremdheit, investissement socio-économique comme marquage identitaire ». Bien qu'il existe différentes catégories d'étrangers ainsi que des formes de relation diverses entre étranger et autochtone dans les sociétés en question, il nous semble justifié de dire, en citant Simmel, que l'étranger est, dans toutes ces sociétés, un élément du groupe même; le statut d'étranger est issu des relations concrètes que l'individu entretient avec les autres membres de ce groupe - l'exemple classique étant la relation jatigi/étranger. Parfois, la tension latente entre cette pluralité vécue, basée sur la différence et la notion de citoyenneté dans l'État-Nation se transforme en conflit - comme nous l'observons actuellement en Côte d'Ivoire.

Dans certains cas, la Fremdheit ne vient pas tant du manque de contact ou même des connaissances réciproques, elle est plus l'expression d'expériences historiques communes (esclavage et colonialisme) et des relations de statut qui en découlent. C'est le cas des Sénégalais à Casablanca. La différence et les frontières ethnico-culturelles sont ressenties par les migrants sénégalais comme un mécanisme d'exclusion voire d'exploitation. Les migrants Wodaabe et Tuareg par contre sont des étrangers au sens propre du terme. Leurs lieux d'origine sont méconnus des populations côtières, et il n'existe entre eux

- contrairement aux relations entre les Sénégalais, les Mauritaniens et les Marocains ni de références communes ni de relations complémentaires. La Fremdheit signifie ici que les fondements des perceptions et des valeurs des partenaires sont différents et que leur contact ne peut pas être considéré comme un processus de médiation ou de négociation - négociation de normes, d'identité, etc. - mais plutôt comme une expérience de la différence.

15 La vie urbaine des migrants Wodaabe en donne un bon exemple. Les camps, qu'ils installent dans les rues de Dakar ou de Bamako, montrent, en mettant en scène le privé et l'intime dans les espaces publics, les limites tenues des échanges entre les populations urbaines et les migrants. En utilisant et organisant l'espace de manière spécifique les Wodabee ne se comportent pas seulement en "étrangers ", mais d'une certaine manière ils se rendent «étrangers » les uns aux autres à l'intérieur de leur 
propre groupe, en renonçant malgré leur proximité aux formes habituelles de comportement social.

Un autre exemple qui montre que "l'étrangeté » peut se mettre en place au sein d'un même groupe est celle des sociétés villageoises du fleuve Sénégal, qui ne se gèrent plus elles-mêmes mais qui sont gérées à distance par des urbains médiateurs entre les villages mêmes et les populations émigrées (Jean Schmitz). C'est leur rôle de gestionnaires des investissements des émigrés et de leurs biens qui confèrent à ces intermédiaires leur pouvoir anciennement dévolu aux anciens des villages.

La notion d'étranger/d'étrangeté peut véhiculer des connotations positives et être le fondement de relations sociales comme le montre le cas des marabouts étrangers à Dakar (Amber Gemmeke). D'une part, leurs clients connotent l'idée « d'étranger » à leur origine rurale et y voient une garantie pour la qualité et l'efficacité des produits qu'ils proposent, alors que d'autre part elle est l'expression d'une distance sociale, laquelle est garante de la confiance que ceux-ci mettent en eux. En même temps les marabouts étrangers deviennent les vecteurs de leur propre "étrangeté " pour des citadins sénégalais et qui plus est pour ceux qui vivent dans les villes européennes et américaines. On retrouve certains aspects de cette notion d'étrangeté dans la société maure avec « ces étrangers » qui sont les témoins intimes de la vie quotidienne, comme les nourrices de la société beydan (Kiki van Til): ici, ce n'est pas l'activité dans le quotidien qui souligne l'étrangeté, mais leur statut même qui en fait des "étrangères » - statut d'esclave, d'ancienne esclave, de descendante d'esclave.

Lors de ce colloque, selon notre discutant Georg Klute, se sont profilées deux thématiques essentielles. Dans la présentation de la migration se dessinent certaines tensions permanentes: d'abord entre les tendances d'émancipation et de libération qui en découle et les résistances au départ et les contraintes au retour, et puis entre l'intime, le quotidien vécu avec l'autre et la distance qui peut s'ériger entre le local et l'étranger. Une proximité extrême mêlée à une distance tout aussi extrême relève des conditions inhérentes de ces relations et dont les modalités seraient encore à étudier. Nos réflexions de départ qui nous ont fait renoncer à l'utilisation du terme de " migration » ont été confirmées en ce sens que la conception même de la « migration » change selon les acteurs, les formes de migrations et aussi selon le lieu de départ.

Un aspect qu'il reste à étudier plus profondément est l'importance de la route utilisée par le migrant, du voyage lui-même et du temps écoulé entre le départ et l'arrivée. Sont-ils le terrain social neutre où se produira le moment de basculement des références aux lieux de départ et d'arrivée, aux normes sociales anciennes et nouvelles auxquelles il faudra s'adapter ou desquelles il faudra se démarquer - sont-ils porteurs d'une (re) construction identitaire? Si c'est le cas, peut-on dire que le processus de migration lui-même est porteur des lieux de destination.

Le second élément qui a dominé est la thématique de la construction identitaire qui dépend des contextes: le lieu d'où l'on vient, là où l'on va, là où l'on arrive. À l'étranger, il s'agira de sauvegarder l'identité de départ, comme pour ces migrants internationaux qui renvoient leurs enfants en vacances dans les lieux d'origine. C'est le lieu d'origine qui est susceptible de transmettre cette identité. Dans les installations à l'étranger, les individus d'un groupe oscilleront en permanence entre une identité collective et une identité individuelle issues des habitudes de comportement des lieux d'origine desquelles découlent les sociabilités qui se mettent en place dans les villes d'installation tant dans les centres urbains occidentaux que saharo sahéliens. 

des anciens esclaves notamment (Florence Boyer), des femmes vis-à-vis des forces familiales autoritaires et des restrictions sociales (Katja Werthmann, Laurence Marfaing, Sophie Caratini). La question qui se pose est de savoir si ces processus d'émancipation résistent au retour. Dans ce contexte il est intéressant de constater que les retombées sur le statut des migrants à leur retour sont entre autre liées à l'accès à l'argent rendu visible dans les lieux de départ grâce aux investissements des migrants. D'autres exemples montrent que la mobilité saisonnière et l'installation dans les villes ne sont qu'une parenthèse dans le cours habituel de la vie des groupes sociaux, c'est le cas des Wodaabe ou encore celui des migrants Tuareg du Niger. La question relative à l'urbanité de ces groupes est à considérer dans les lieux d'arrivée. Notamment la manière dont les migrants "imprègnent " leurs lieux d'installation; ce phénomène semble lié à la durée de l'installation qui engendre la relation avec les groupes locaux. A contrario, il semble que la non-installation puisse être un choix, parfois une stratégie mise en place par les migrants eux-mêmes.

Ce colloque a montré que se concentrer sur une région définie et sur des conditions de mobilités historiques et socio-culturelles spécifiques permet une investigation comparative de certains aspects : par exemple, celui du fondement et des fonctions des différents modes d'installation et de la signification de l'extranéité culturelle. Les discussions ont mis aussi l'accent sur la problématique des niveaux de l'analyse et de la relation entre les orientations et les développements individuels et collectifs. Dans cette perspective, Jean Schmitz a présenté une observation « mesoscopique » basée sur une approche de réseaux familiaux, Florence Boyer a traité des conditions spatiales de l'émancipation individuelle, Elisabeth Boesen du caractère collectif de la mobilité qui comporte en même temps une individuation et enfin Kiki Van Til des neighbourhoods.

On mentionnera aussi le recours à la notion de "projet migratoire». Dans cette logique, Florence Boyer propose une approche processive et la liaison des différents niveaux de l'analyse (celui de l'individu, de la famille, du groupe...). Cette démarche a des conséquences sur la pratique du terrain dans la mesure où l'accompagnement des migrants, le voyage en commun, devient une nécessité. Par contre, comme l'a rappelé J. Schmitz, certains chercheurs estiment qu'en matière d'étude de la migration, il est indispensable de se concentrer sur les sociétés d'origine, comme cela est le cas depuis une dizaine d'années dans les études sur les migrations africaines.

\section{(Liste des participants et titres des communications)}

- Linda Beck (Columbia University, New York) : A Comparative Analysis of Translocality between and among the Hal-Pulaaren and Murid Communities New York City.

- Daouda Gary (Université Denis Diderot, Paris 7): Migrations de la sécheresse et/ou de la pauvreté. Les migrations en provenance du Sahel vues de la Côte d'Ivoire.

- Abdoulaye Kane (University of Florida) : De Medina Gounass à Fez: mobilité, sociabilité et échange autour de la Tijaniyya.

- Gregory Mann (Columbia University, New York): Des étrangers bien connus: logiques migratoires et statuts politiques entre l'Afrique francophone et la France (années 1960).

- Florence Boyer (Migrinter, Université de Poitiers) : Exodants-esclaves de Bankilaré (Niger) à Abidjan : la libération par l'urbain ou par le mouvement.

- Sophie Caratini (Laboratoire CITERES, Université de Tours) : La prison du temps ou l'arrêt des nomades : les camps de réfugiés sahraouis de la hamada de Tindouf. 
- Dida Badi (CNRPAH, Alger) : Le rôle des communautés sahéliennes dans l'économie locale d'une ville saharienne : Tamanrasset (Sahara algérien).

- Andre Bourgeot (CNRS, Paris) : Urbanisation, mouvement, sédentarisation des populations et mobilité des capitaux. La culture de l'oigon en Air (Niger).

- Isaie Dougnon (Université de Bamako): Kayakaya ou migrants itinérants Songhay: l'émergence d'une identité New Worker au Ghana.

- Laurence Marfaing (ZMO, Berlin) : Commerce, constructions spatiales et relationnelles dans un espace urbain : commerçantes sénégalaises à Casablanca.

- Benedetta Rossi (SOAS, London) : Status and mobility in the Ader Region of Niger.

- Marco Scholze (Université de Bayreuth) : «Agadez nous appartient !» Relations et conflits entre les acteurs urbains et mobiles dans le tourisme saharien.

- Katja Werthmann (Universität Mainz) : Dans un monde masculin : le travail de femmes dans un camp de chercheurs d'or au Burkina.

- Elisabeth Boesen (ZMO, Berlin) : Des localités nomades : les «maisons » Wodaabe en brousse et en ville.

- Amber Gemmeke (Leiden University) : The Map of Magic. Marabouts in suburban Dakar.

- Kiki van Til (Leiden University): Nomadic pastoralists and neo-townspeople: (re) construction of identity and social relationships in a small Mauritanian town.

- Jean Schmitz (EHESS, Paris) : Gouvernance et espace tiers : la communauté transnationale des gens du fleuve Sénégal.

\section{BIBLIOGRAPHIE}

Adepoju, Aderanti (1995) Migration in Africa. An overview. In : Baker, Jonathan \& Tade Akin Aina (Hg.), The migration experience in Africa. Uppsala : Nordiska Afrikainstitutet, pp. 87-109.

Berland, Joseph C. (1979) Peripatetic, pastoralist and sedentist interaction in complex societies. Nomadic peoples Newsletter $4: 6-8$.

Boyer Florence (2005) Le projet migratoire de migrants touaregs de la zone de Bankilaré : la pauvreté désavouée. Stichproben. Wiener Zeitschrift für kritische Afrikastudien 5 : 47-67.

Bredeloup Sylvie (2003) Le migrant africain et la ville. In : Coquéry-Vidrovitch, Catherine et al. (éds.), Être étrangers et migrant en Afrique au XX siècle. Enjeux identitaires et modes d'insertion. Vol. II : Dynamiques migratoires, modalités d'insertion urbaine et enjeux d'acteurs. Paris : L'Harmattan, pp. 53-61.

Dijk, Han van et al. (2001) Population mobility in Africa. In : de Bruijn, Mirjam et al (Hg.), Mobile Africa. Changing patterns of movement in Africa and beyond. Leiden : Brill, pp. 9-26.

Fortes, Meyer (1975) Strangers. In : Ders. \& Sheila Patterson (Eds.), Studies in African social anthropology. London : Academic Press, pp. 229-253.

Gervais-Lambony, Philippe (2003) Migrants et citadins dans les villes africaines. Éléments de réflexion à partir de quelques cas (Afrique du Sud, Zimbabwe, Togo). In : Coquery-Vidrovitsch, Catherine et al. (Eds.), Être étranger et migrant en Afrique au XX $\mathrm{X}^{e}$ siècle. Enjeux identitaires et modes 
d'insertion. Vol. II : Dynamiques migratoires, modalités d'insertion urbaine et enjeux d'acteurs. Paris :

L'Harmattan, pp. 17-30.

Olofson, Harold (1976) Yawon dandi : A Hausa category of migration. Africa 46 : 66-79.

Rao, Aparna (1985) Des nomades méconnus. Pour une typologie des communautés péripatétiques. L'Homme 95 (3) : 97-120.

Simmel, Georg (1968) (1908) Der Raum und die räumlichen Ordnungen der Gesellschaft. In : Ders., Soziologie. Untersuchung über die Form der Vergesellschaftung. Berlin : Duncker und Humboldt, pp. 460-526.

Spittler, Gerd (2001) Teilnehmende Beobachtung als dichte Teilnahme. Zeitschrift für Ethnologie $126: 1-25$.

Vianna, Pedro (1999) Les défis de l'intégration dans une société médiatique. Migration Société 11 (n66, nov.-déc.) : 99-107. 